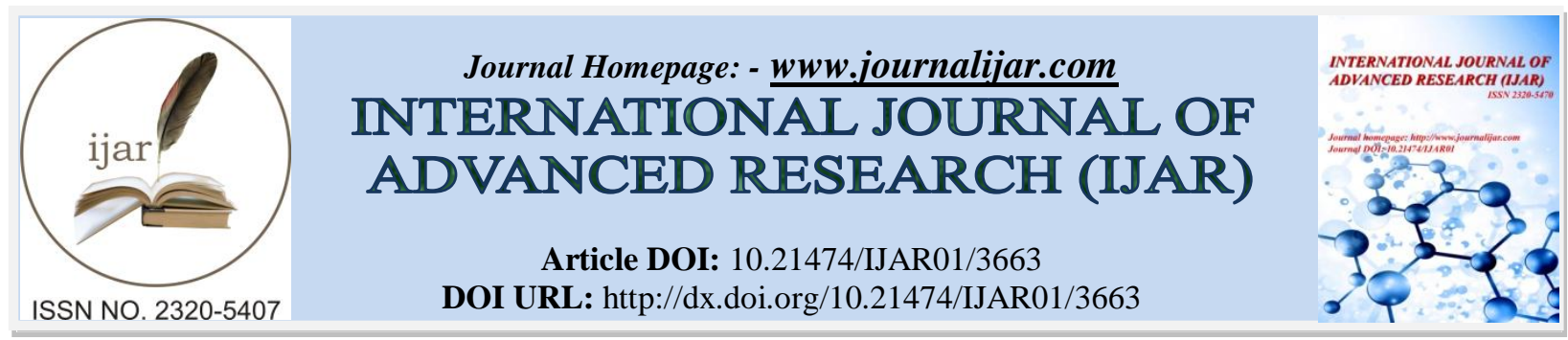

RESEARCH ARTICLE

\title{
THEORETICAL STUDY OF THE EFFECT OF LINEAR DEFORMATION ON BULK MODULUS AND COMPRESSIBILITY OF METALS.
}

*Adesakin G. E.

Department of Physics, Ekiti State University, Ado-Ekiti, Nigeria.

\section{Manuscript Info}

Manuscript History

Received: 10 January 2017

Final Accepted: 10 February 2017

Published: March 2017

Key words:-

Deformation, Bulk Modulus,

Compressibility, Collision,

Pseudopotential Model, Thermal

Resistivity, Electronic Concentration.

\begin{abstract}
The effects of deformation on the bulk modulus and compressibility of different elemental metals were computed and studied based on pseudopotential formalism. The electron density parameters of deformed metals under the application of different strains were obtained for different metals. The poison ratio relating the transversal compression to elongation in the direction of applied deformation for different elemental metals were computed using elastic moduli for homogeneous isotropic material and used in this work. The results obtained revealed that there is a good agreement between the computed and experimental value of the bulk modulus and compressibility of metals. There is high concentration of electron in the high density region than in the low density region for the bulk modulus and compressibility of metals these seems to suggest that the bulk modulus and compressibility of metals depend on the density of valence electron in metals. The bulk modulus of all the metals investigated decreases as deformation increases. These could be due to reduction in electron compressible rate, fracture density and increase in inter atomic distance between the electrons in the metals. The effect of deformation is more pronounced on the polyvalent metals than in alkaline metals these could be due to the high electronic concentration and high electronic energy level of the free atom in the alkaline metal. Compressibility increases with an increase in deformation for all the metals investigated. These could be due to an increase in the collision between the interacting electrons in metals which forces the compressibility of the electron in metals to increase as deformation increases.
\end{abstract}

Copy Right, IJAR, 2017,. All rights reserved.

\section{Introduction:-}

The simplest model for describing electrons in solid is to assume that the valence electrons of an atom in solids are free to move anywhere throughout the volume of the material but are not allowed to leave (Pillai, 2010). The electric and magnetic properties of solids are mainly determined by the properties of electrons in them, the energy levels of electrons hold the key to the properties of solids (Solymar and Walsh, 1984). The electrons in solid behave as a delocalized gas of free electrons (Elliot, 1999).

Corresponding Author:- Adesakin G. E. Address:- Department of Physics, Ekiti State University, Ado-Ekiti, Nigeria. 
Pseudopotential approach in a way gives organizing experimental and computational information about conduction electrons in solid in a compact and physical form (Marder, 2000). The goal of pseudopotential theory is to obtain the key physical properties of solids by dealing with only the valence electrons (Kiejna, 1993). Transport processes in solids leads to electrical conductivity, thermal conductivity, thermal resistivity etc. These are affected by electronelectron, electron-impurity and electron-phonon scattering. Carriers in conductors move in a definite direction under applied fields and temperature gradients (Duan and Guojun, J.2005)

The bulk modulus of solid is a measure of its resistance to uniform compression and is defined as the ratio of the infinitesimal pressure increase to the resulting relative decrease in volume (Animalu, 1977). Bulk modulus is a thermodynamic quantity, and is specify by how the temperature varies during compression (Kittel, 1976). Compressibility is determined by the second derivative of the total energy with respect to the volume and can also be express as the reciprocal of the bulk modulus of solids (Kukkonen and Wilkins, 1979). The compressibility sum rule expresses the self-consistency condition that the static response of the system to the long-wavelength perturbation is equal to the compressibility where the density dependent ground state energy obtained via the Monte Carlo method is used to determine the compressibility via thermodynamic relations. (Iwamoto, 1999).

Deformation is the change in shape or size of solid (Backofen, 1972). A solid body is deform when a stress is applied to it, all solid materials exhibit nearly Hookean behaviour for small enough strain or stress (Borg,1990). When a material is subjected to external forces its behaviour depends not only on the magnitudes of the forces and the inherent strength of the material itself but also on the way the forces are applied and combined. The particular combination of forces may cause the material to deform elastically or plastically. The amount of deformation depends on knowing the intensity of the forces at all points throughout the material (Hugh Ford and Alexander, 1977). Metals can be deformed by compressive, tensile and torsion force. During deformation, atoms at the surface and interior of metals changes together with their atomic distance depending on the metallic surface area that is subjected to different deformation. The contact potential difference on the metal surface also changes during deformation (Borg, 1990).

Over the years, the bulk modulus and the electron compressibility of solids has been studied both theoretically and experimentally. Osiele and Edenma, (2009) developed a model for computing the bulk modulus of metals based on the theory of structureless pseudopotential. The results obtained revealed that the calculated and experimental bulk modulus of metals varies in the same manner with experimental values. Ling and Gelatt(1981) used a local pseudopotential constructed from the bulk stability condition alone to calculate the shear and bulk moduli of 19 simple metals. They also studied the chemical trends in the elastic modulus of the simple metals and calculated the ratio of shear modulus to bulk modulus, the result obtained was in good agreement with experimental value. Vackar et al, (1998) developed the all-electron pseudopotential model and used it to calculate the lattice constant and bulk modulus of silicon, diamond, non-magnetic cobalt, cubic TiC and hexagonal TiS. The results they got were in satisfactory agreement with experimental values. Osiele et al (2011) develop a model for computing thermal resistivity, compressibility ratio and screening parameter of metals based on free electron theory. The result obtained revealed that thermal resistivity of metals increases with an increase in the electron gas parameter. While the compressibility ratio of metals decreases with an increase in the electron gas parameter. On the screening parameter, the thermal resistivity also increases with an increase in the screening parameter while the compressibility ratio decreases with an increase in the screening parameter. Keijna and Pogosov, (2000) experimentally investigate the effect of deformation on electronic properties of some metals by measuring directly a sample of deformed metal using kelvin method. They observed that the contact potential difference of metals decreases when tensed and increases when compressed. Adeshakin and Osiele (2012) computed the surface energy and surface stress of deformed metals based on the structureless pseudopotential formalism. The results obtained revealed that deformation causes a reduction of surface energy and this reduction is more pronounced in simple and alkaline metals. Tensile stress is present in most metallic surfaces whose surface stress were computed, although a few metals possess compressive stress on their surfaces. In the presence of deformation, the surface stress of some metals decreases, while deformation causes an increase in the surface stress of some metals. Adesakin, (2016) develop a model to compute the electrical conductivity of different elemental metals based on pseudopotential formalism. The results obtained revealed that there is a good agreement between the computed and experimental value of the electrical conductivity of metals. There is high concentration of electron in the high density region than the low density region. The electrical conductivity of metals decreases as deformation (strains) increases for all the metals investigated. The effect of deformation is more pronounced on the electrical conductivity of noble and transition metals than in alkaline metals. 
In this work the structureless pseudopotential is extended to the study of the effects of linear deformation on the Bulk modulus and compressibility of some metals. This will give an insight into how the Bulk modulus and compressibility of metals changes with deformation. The metals were chosen based on their technological and industrial applications and availability of some physical constants of metals that is required for computation.

\section{Theory And Calculations:-}

Electrons are quantum not classical particles and so the allowed electronic states must be the solutions of the Schrodinger equation which in the general (non-relativistic) case where the potential energy $\mathrm{V}$ is a function of both space and time is:

$$
\frac{\hbar^{2}}{2 m_{e}} \nabla^{2} \psi(r, t)+V(r, t) \psi(r, t)=i \hbar \frac{\partial}{\partial t}[\psi(r, t)]
$$

Where $m_{e}$ is the electron mass, $\hbar$ is the normalised Planck's constant and $\psi(r, t)$ is the electron wavefunction.

The energy functional of the system of interacting electron in an external potential arising from the interaction with ions represented by a local pseudopotential $\phi$ can be expressed as a functional of the valence electronic density as

$$
\begin{aligned}
E[n]=T_{s}[n]+ & E_{x c}[n]+\frac{1}{2} \int d^{3} r \int d^{3} r^{\prime} \frac{n(r) n\left(r^{\prime}\right)}{\left|r^{\prime}-r\right|}+\int d^{3} r \phi\left(\left|r-R_{i}\right|\right) n(r) \\
& +\frac{1}{2} \sum_{i} \frac{Z^{2}}{\left|R_{i}-R_{j}\right|}
\end{aligned}
$$

where the first three terms represents the kinetic, exchange-correlation and electrostatic energy of the interacting system. The two last terms describes the interaction of electrons with the ions at sites $R_{i}$ via a pseudopotential, $\phi$ and the Coulomb interaction between the ions.

The total energy required to assemble the valence electrons and ions to form the solid which is the binding energy is

$$
E=T_{s}(n)+E_{x c}+W_{R}+E_{m}
$$

Where $T_{s}$ is the kinetic energy, $E_{x c}$ is the sum of the exchange and correlation energies, $W_{R}$ is the average value of the non-Coulombic part of the pseudopotential and $E_{m}$ is the Madelung energy of points ions embedded in a uniform negative background.

Based on the density functional theory in the low density limit, the kinetic and exchange energies are given as (Kiejna and Wojciechowski, 1996)

$$
T_{s}=\frac{1.105}{r_{s}^{2}} \text { and } E_{x c}=\frac{-0.458}{r_{s}}
$$

Where $r_{s}$ is the electron density parameter, which is describe as the radius of a sphere containing one electron on the average.

The correlation energy used in this work is that of Carperly and Alder as parameterised by Perdew and Zunger and is given as

$$
E_{c}=\frac{-0.1423}{1+1.0529 r_{s}^{2}+0.3334 r_{s}}
$$

From equation (2.3), the binding energy is

$$
E=\frac{1.105}{r_{s}^{2}}-\frac{0.458}{r_{s}}-\frac{0.1423}{1+1.0529 r_{s}^{2}+0.3334 r_{s}}+W_{R}+E_{m}
$$

The average value of the repulsive part of the pseudopotential is

$$
W_{R}=\frac{n}{2} \int d r W_{R}(r)=\frac{n_{a v}}{2} \int 4 \pi r^{2} \frac{Z}{r} d r=2 \pi n_{a v} r_{c}
$$

Where $n_{a v}$ is the average density of the electron.

The self-energy of the electrostatic interaction average over the whole Wigner-Seitz sphere is

$$
E_{e s}=\frac{\frac{1}{2} \int V(r) 4 \pi r^{2} d r}{\frac{4 \pi r_{0}{ }^{3}}{3}}=\frac{3 Z}{5 r_{0}}
$$

The factor of half is to avoid double counting. The positive contributions are compensated for by the negative energy of the electrons interacting with the uniform positive background. 
The Medelung energy for a jellium system is given by (Perdew and Zunger, 1981) as

$$
E_{m}=\frac{-3 Z}{2 r_{0}}+E_{e s}=\frac{-9 Z}{10 r_{0}}
$$

Where $r_{0}=Z^{1 / 3} r_{s}$. Substituting equation (2.7) and (2.9) into (2.8), the binding energy according to the structureless pseudopotential model is obtain as

$$
E=\frac{1.105}{r_{s}^{2}}-\frac{0.458}{r_{s}}-\frac{0.1423}{1+1.0529 r_{s}^{2}+0.3334 r_{s}}+2 \pi n r_{c}-\frac{9 Z}{10 r_{0}}
$$

But $r_{0}=Z^{1 / 3} r_{s}$, and $n=4 \pi r_{s} / 3$, hence

$$
E=\frac{1.105}{r_{s}^{2}}-\frac{0.458}{r_{s}}-\frac{0.1423}{1+1.0529 r_{s}^{2}+0.3334 r_{s}}+\frac{3 r_{c}{ }^{2}}{2 r_{s}{ }^{3}}-\frac{9 z^{2} / 3}{10 r_{s}}
$$

Where $r_{s}$ is the electron density parameter of metals and $r_{c}$ is the Ashcroft core radius given as (Perdew,et, al, 1990)

$$
r_{c}=\left[\frac{-2}{15}\left(\frac{9 \pi}{4}\right)^{2 / 3} r_{s}+\frac{1}{6 \pi}\left(\frac{9 \pi}{4}\right)^{1 / 3} r_{s}^{2}+\frac{1}{5} Z^{2 / 3} r_{s}^{2}+\frac{2}{9} r_{s}^{4} \frac{d E_{c}}{d r_{s}}\right]^{1 / 2}
$$

Where $E_{c}$ is the correlation energy given by equation (2.5)

Since the conduction electrons in a solid is being treated as a gas of particles, albeit a quantum gas, they will exert a pressure given at zero kelvin by (Pillai, 2010)

$$
P=-\left(\frac{\partial E}{\partial V}\right)_{N}
$$

Since it is assumed that the potential energy is zero, the origin of the pressure is regarded as being due to the repulsion experienced by electrons caused by the Pauli Exclusion Principle, when they are compressed and tend to occupy the same region space.

The bulk modulus which measures resistance to structure preserving volume-changing for an underformed metal is (Ling and Gelatt, 1981)

$$
B=-V\left(\frac{\partial p}{\partial v}\right)_{T, N}=\frac{1}{12 \pi}\left(\frac{1}{r_{s}^{2}} \frac{\partial^{2} E}{\partial r_{s}^{2}}-\frac{2}{r_{s}^{2}} \frac{\partial E}{\partial r_{s}}\right)
$$

While compressibility for an undeformed metals is obtained as

$$
\tau=\frac{1}{\frac{1}{12 \pi}\left(\frac{1}{r_{s}^{2}} \frac{\partial^{2} E}{\partial r_{s}^{2}}-\frac{2 \partial E}{r_{s}^{2} \partial r_{s}}\right)}
$$

Considering only the kinetic energy of the free-electron for which $P \propto V^{-5 / 3}$, the zero kelvin electronic contribution to the bulk modulus is (Elliott, 1997)

$$
B_{K E}=\frac{0.586}{r_{s}^{5}}(a . u)
$$

The compressibility of the uniform electron gas is determined by the second derivative of the total energy with respect to volume

$$
K=\left(V \frac{\partial^{2} E}{\partial V^{2}}\right)^{-1}
$$

The compressibility of the ratio of the non-interacting electron gas $K_{\text {free }}$ to that of the interacting electron gas $\mathrm{K}$ is given as (Bowen Etal, 1974)

$$
\frac{K_{\text {free }}}{K}=\left[1-\frac{4}{\pi} \propto r_{s} \frac{1}{4} \frac{\pi \alpha}{24} r_{s}^{5} \frac{d}{d r_{s}}\left(\frac{1}{r_{s}^{2}} \frac{d E_{c}}{d r_{s}}\right)\right]
$$

Where $r_{s}$ is the electron density parameter, $\propto=(4 / 9 \pi)^{1 / 3}$ and $E_{c}$ is the correlation energy per electron which is given by equation (2.5)

For a deformed metal, the average electron density parameter of metal is

$$
r_{s u}=r_{s}\left[1+(1-2 v) U_{x x}\right]^{1 / 3}
$$

where $v$ is the Poisson ratio relating the transversal compression to elongation in the direction of applied deformation and $U_{x x}$ is the uniaxial strain.

The bulk modulus and the compressibility of the deformed solid at absolute zero is given by

$$
\beta=\frac{1}{12 \pi}\left(\frac{1}{r_{s u}^{2}} \frac{\partial^{2} E}{\partial r_{s u}^{2}}-\frac{2}{r_{s u}^{2}} \frac{\partial E}{\partial r_{s u}}\right)
$$


and

$$
\mathrm{\gamma}=\frac{1}{\beta}=\frac{1}{\frac{1}{12 \pi}\left(\frac{1}{r_{s u}{ }^{2}} \frac{\partial^{2} E}{\partial r_{s u}{ }^{2}}-\frac{2}{r_{s u}{ }^{2}} \frac{\partial E}{\partial r_{s u}}\right)}
$$

This study generalized the work of Kiejna and Pogosov (2000) due to the shortcomings of the electron density parameter of deformed metals. They failed to account for metal dilation by assuming a constant value for the Poisson ratio of metals which leads to neglect of the uniaxial strain in their computation. In this work, the Bulk modulus and electron compressibility of deformed metals were computed using equation (2.20) and (2.21) and how deformation affects these properties of metals is studied. Also the variations of Bulk modulus and electron compressibility with electron density parameters were computed and studied using equation (2.14) and (2.15).

\section{Results And Discussion:-}

Figure 1 shows the variation of bulk modulus with electron density parameter for some monovalent, divalent, trivalent and polyvalent metals. Figure 1 revealed that there is high concentration of electron in the high density region than the low density region for both computed and experimental values of the bulk modulus. These seem to suggest that the higher the density of valence electron and the electronic concentration of metal the higher the bulk modulus and the lower the density of valence electron and electronic concentration of metal the lower the bulk modulus. In the high density region we have the alkaline metals and in the lower density region we have the noble and inner transition metals. Although, the bulk modulus of metals in figure 1 seems not to exhibit a particular trend and these could be due to the fact that there are some basic properties that contribute to the bulk modulus of metals that the model does not take into consideration such as the nature of chemical bonding, impurity atom and atomic size of the metals. The trend exhibited by metals in figure 1 revealed that there is an agreement between the computed and experimental values of the bulk modulus. This is more pronounce in some of the metals the high density region. The experimental value of the bulk modulus used in these work is obtained from solid state Physics by Kittel (1976). Figure 2 shows the variation of compressibility with electron density parameter for group one, group two, group three and transition metals. The trend exhibited by metals in figure 2 seems to be linear and exhibit a neither increase nor decrease in compressibility for metals the high density region but towards where $r_{s} \geq 2.75$ a.u there is a clear increase in the compressibility of the metals as we go from one metals to another. The trend exhibited by metals in figure 2 shows that compressibility is truly the inverse of bulk modulus. Figure 2 revealed that metals in the high density region have low compressibility while metals in the low density region have high compressibility. These could be due to the variable valence electron contribution in the solid and the nature of their electronic state structure.

Figure 3 shows the variation of bulk modulus with deformation for some monovalent, divalent, trivalent and polyvalent metals. Figure 3 revealed that the bulk modulus of all the metals investigated decreases with an increase in deformation. These could be due to reduction in electron compressible rate, fracture density and the pressure increase during deformation. The trend exhibited by metals in figure 3 shows that potassium has the lowest bulk modulus while molybdnum were found to be having the highest bulk modulus among all the metals subjected to different deformation. These could be due to the high electronic concentration, high equilibrium distance between the electron and high electronic energy level of the free atom in the alkaline metal that cause the bulk modulus of potassium to be the least affected among all the metals subjected to different deformation. Figure 3 also revealed that metals in the high density region has high bulk modulus while metals in the low density region were found to be having low bulk modulus among all the metals subjected to different deformation. These seems to suggest that as deformation increase the pressure and the atomic volume of the metals increases and thereby reduces the strength of interaction between the electron which forces the bulk modulus of the metals to decrease as deformation increases. Furthermore, the trend exhibited by metals in figure 3 revealed that as deformation increases, there is an increase in the electron temperature due to delocalisation as these may cause a decrease in some of the elastic properties that the bulk modulus of metals depend upon.

Figure 4 shows the variation of compressibility with deformation for some metals containing group one, group two, group three and transition metals. Figure 4 revealed that the electron compressibility increase with an increase in deformation for all the metals investigated. These seems to suggest that as deformation increases the collision and temperature between the interacting electron in metals increases and their by forces the compressibility of the electron in metals to increase as deformation increases. Figure 4 also revealed that metals in the high density region has low compressibility while metals in the low density region has high compressibility, these could be due to the 
change in the electronic energy level of the free atoms in the solid that causes the number of valence electrons per unit cell to occupy more sites during deformation which result to an increase in the compressibility as deformation increases. Among all the metals subjected to different deformation, potassium has the highest compressibility; this could be due to the high density of the free valence electron, compression factor and electronic concentration in the metal. The trend exhibit by metals in figure 3 and 4 revealed that the bulk modulus and compressibility of metals is strongly affected by deformation.

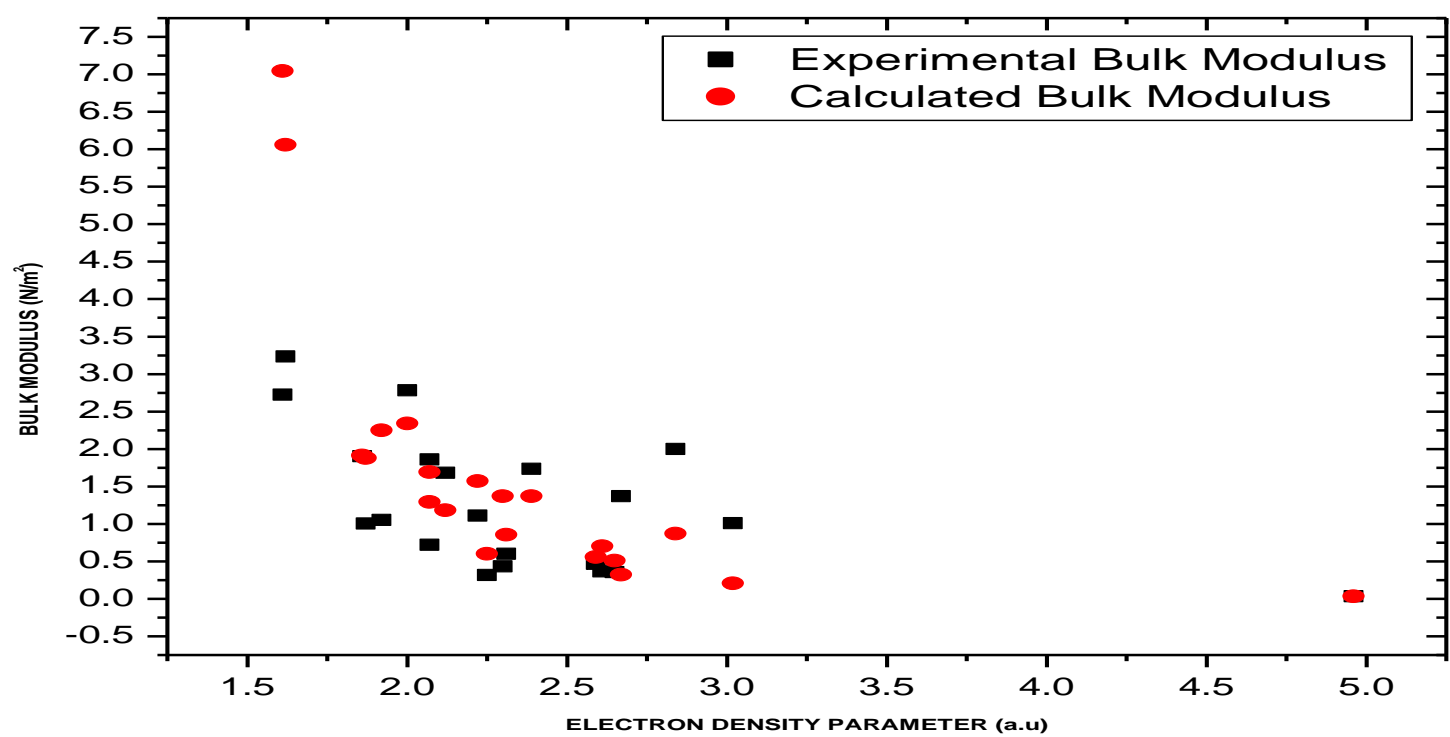

Figure 1:-Variation of Bulk Modulus with Electron Density Parameter for Undeformed Metals.

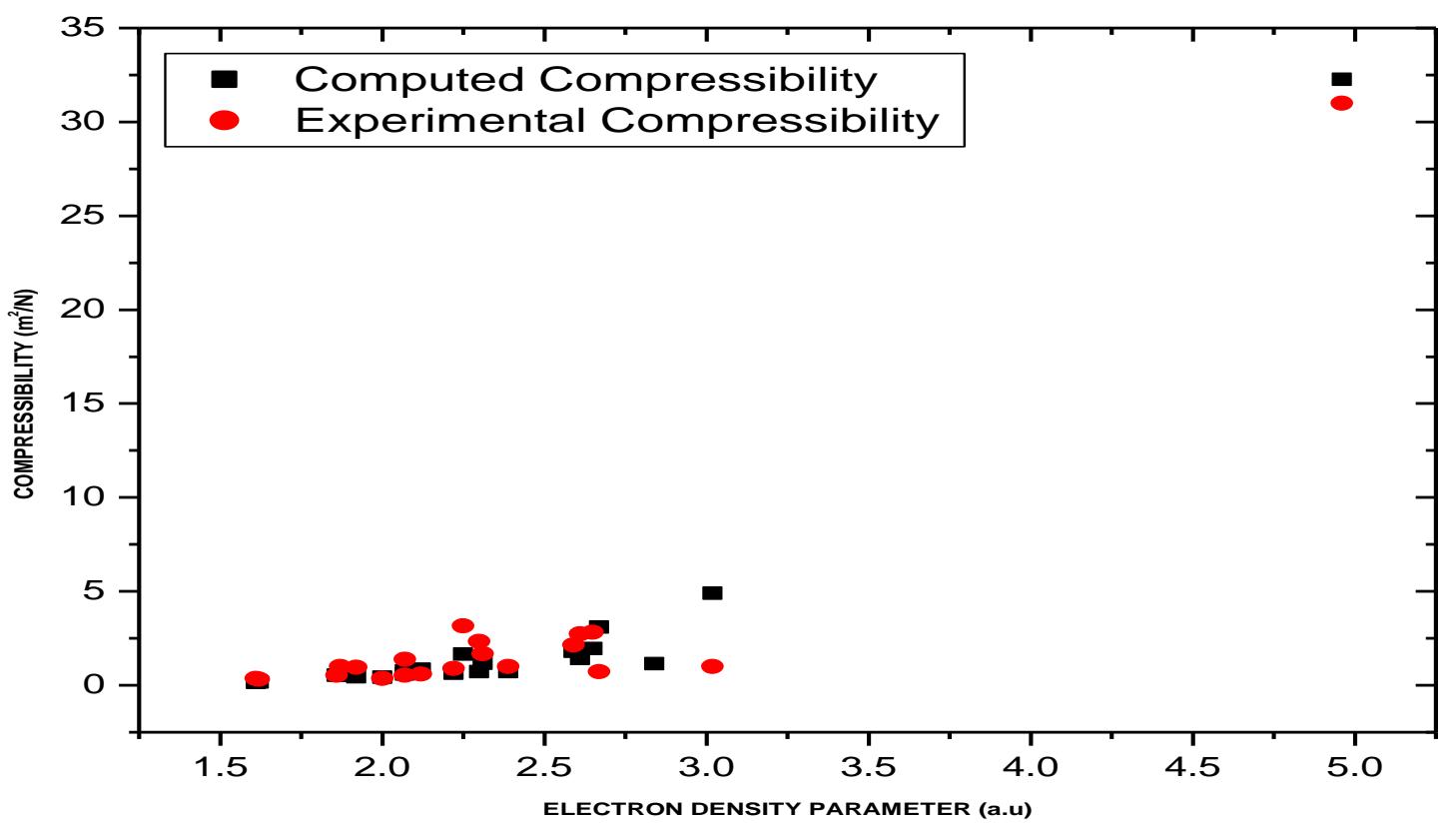

Figure 2:- Variation of Compressibility with Electron Density Parameter for Undeformed Metals 

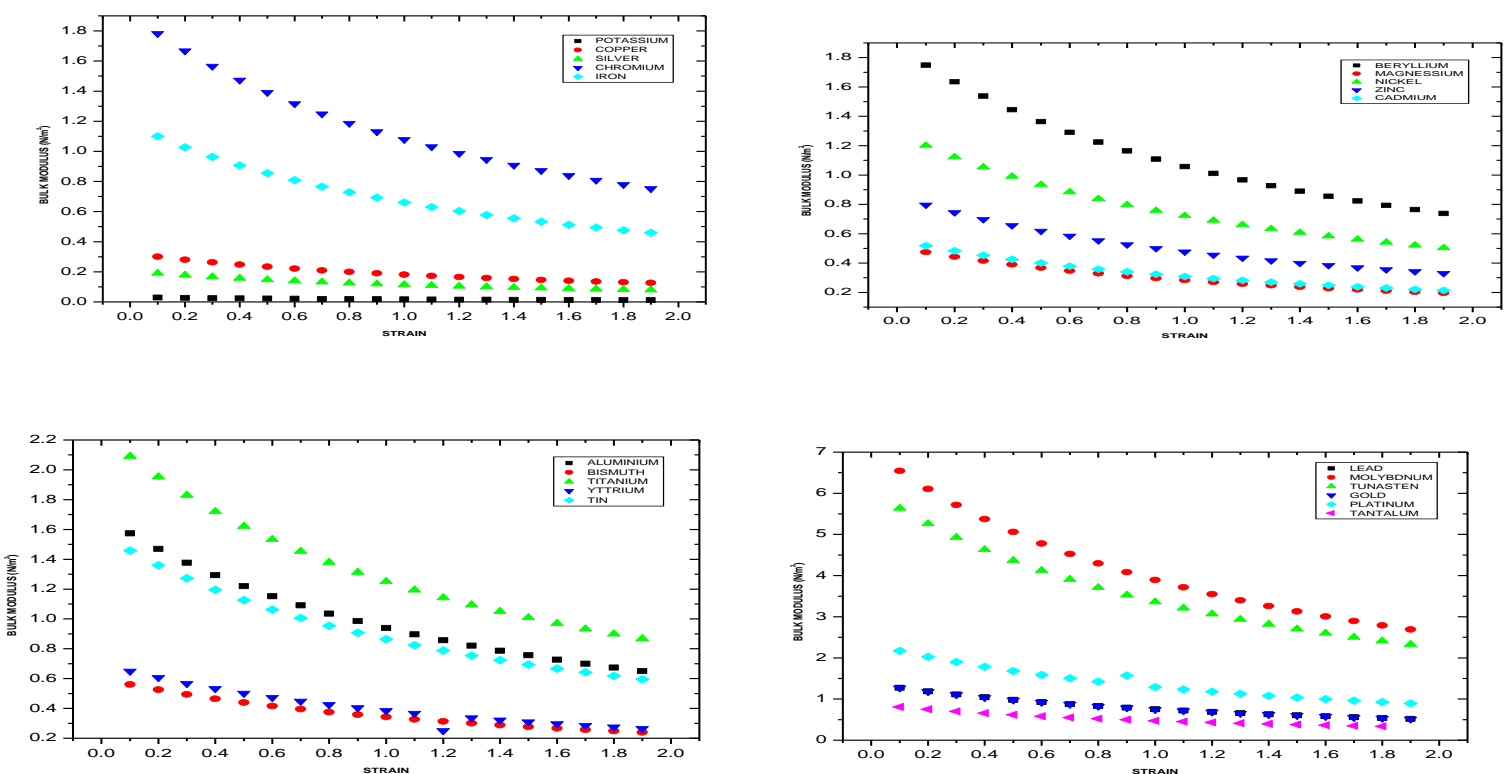

Figure 3:- Variation of Bulk Modulus with Deformation for some Metals
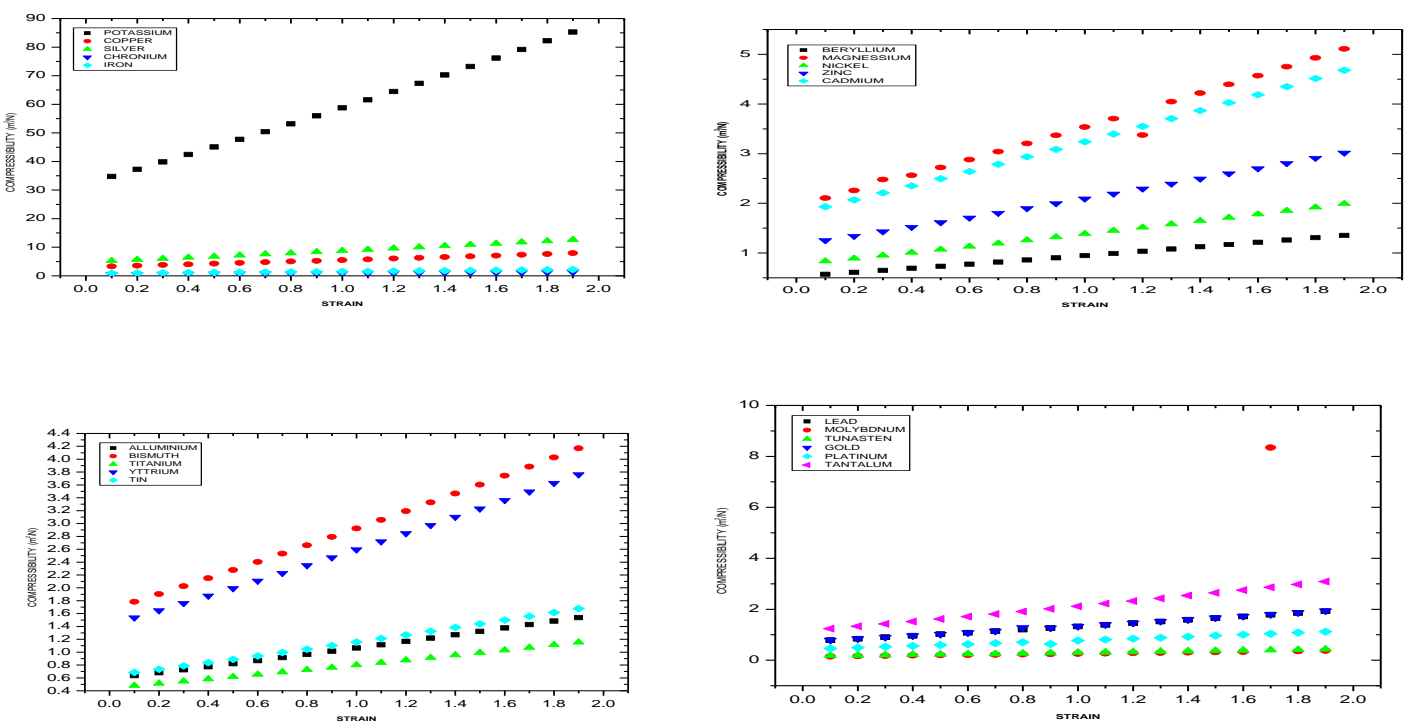

Figure 4:- Variation of Compressibility with Deformation for Some Metals. 
Table 1: Bulk modulus of Deformed Metals $\left(\mathrm{Nm}^{-2}\right)$

\begin{tabular}{|l|l|l|l|l|l|l|l|l|l|l|}
\hline & \multicolumn{9}{|c|}{ Strain } & \multicolumn{9}{|l|}{$\mid$} \\
\hline $\mathrm{Metals}$ & $\mathrm{r}_{\mathrm{s}}$ (a.u) & 0.2 & 0.4 & 0.6 & 0.8 & 1.0 & 1.2 & 1.4 & 1.6 & 1.8 \\
\hline $\mathrm{K}$ & 4.96 & 0.0268 & 0.0236 & 0.0210 & 0.0188 & 0.0170 & 0.0155 & 0.0142 & 0.0131 & 0.0122 \\
\hline $\mathrm{Cu}$ & 2.67 & 0.281 & 0.248 & 0.221 & 0.200 & 0.181 & 0.166 & 0.152 & 0.141 & 0.131 \\
\hline $\mathrm{Ag}$ & 3.02 & 0.178 & 0.157 & 0.140 & 0.126 & 0.114 & 0.104 & 0.0958 & 0.0884 & 0.0821 \\
\hline $\mathrm{Be}$ & 1.87 & 1.636 & 1.445 & 1.291 & 1.164 & 1.058 & 0.967 & 0.890 & 0.823 & 0.765 \\
\hline $\mathrm{Mg}$ & 2.65 & 0.443 & 0.390 & 0.347 & 0.312 & 0.283 & 0.258 & 0.237 & 0.219 & 0.203 \\
\hline $\mathrm{Cr}$ & 1.86 & 1.688 & 1.474 & 1.317 & 1.187 & 1.079 & 0.987 & 0.908 & 0.840 & 0.780 \\
\hline $\mathrm{Fe}$ & 2.12 & 1.027 & 0.906 & 0.808 & 0.728 & 0.661 & 0.604 & 0.555 & 0.513 & 0.476 \\
\hline $\mathrm{Ni}$ & 2.07 & 1.123 & 0.991 & 0.884 & 0.796 & 0.723 & 0.661 & 0.607 & 0.561 & 0.521 \\
\hline $\mathrm{Zn}$ & 2.31 & 0.745 & 0.656 & 0.585 & 0.526 & 0.477 & 0.436 & 0.401 & 0.370 & 0.343 \\
\hline $\mathrm{Cd}$ & 2.59 & 0.483 & 0.425 & 0.379 & 0.341 & 0.309 & 0.282 & 0.259 & 0.239 & 0.222 \\
\hline $\mathrm{Al}$ & 2.07 & 1.469 & 1.294 & 1.152 & 1.036 & 0.939 & 0.857 & 0.787 & 0.727 & 0.674 \\
\hline $\mathrm{Bi}$ & 2.25 & 0.525 & 0.465 & 0.416 & 0.376 & 0.342 & 0.313 & 0.289 & 0.267 & 0.248 \\
\hline $\mathrm{Ti}$ & 1.92 & 1.952 & 1.720 & 1.533 & 1.379 & 1.251 & 1.142 & 1.049 & 0.969 & 0.899 \\
\hline $\mathrm{Y}$ & 2.61 & 0.607 & 0.533 & 0.474 & 0.426 & 0.385 & 0.351 & 0.322 & 0.297 & 0.276 \\
\hline $\mathrm{Sn}$ & 2.22 & 1.360 & 1.195 & 1.061 & 0.594 & 0.864 & 0.788 & 0.723 & 0.667 & 0.618 \\
\hline $\mathrm{Pb}$ & 2.30 & 1.187 & 1.043 & 0.927 & 0.832 & 0.754 & 0.687 & 0.630 & 0.581 & 0.539 \\
\hline $\mathrm{Mo}$ & 1.61 & 6.107 & 5.372 & 4.781 & 4.296 & 3.892 & 3.551 & 3.259 & 3.008 & 2.789 \\
\hline $\mathrm{W}$ & 1.62 & 5.260 & 4.630 & 4.123 & 3.707 & 3.360 & 3.067 & 2.817 & 2.600 & 2.412 \\
\hline $\mathrm{Au}$ & 2.39 & 1.183 & 1.039 & 0.923 & 0.828 & 0.749 & 0.683 & 0.626 & 0.577 & 0.535 \\
\hline $\mathrm{Pt}$ & 2.00 & 2.028 & 1.784 & 1.587 & 1.426 & 1.292 & 1.179 & 1.082 & 0.998 & 0.926 \\
\hline $\mathrm{Ta}$ & 2.84 & 0.750 & 0.658 & 0.584 & 0.523 & 0.473 & 0.431 & 0.394 & 0.363 & 0.336 \\
\hline
\end{tabular}

Table 2: Compressibility of Deformed Metals $\left(\mathrm{m}^{2} \mathrm{~N}\right)$

\begin{tabular}{|l|l|l|l|l|l|l|l|l|l|l|}
\hline & \multicolumn{9}{|c|}{ Strain } \\
\hline Metals & $\mathrm{r}_{\mathrm{s}}(\mathrm{a} . \mathrm{u})$ & 0.2 & 0.4 & 0.6 & 0.8 & 1.0 & 1.2 & 1.4 & 1.6 & 1.8 \\
\hline $\mathrm{K}$ & 4.96 & 37.259 & 42.419 & 47.725 & 53.169 & 58.742 & 64.439 & 70.253 & 76.179 & 82.212 \\
\hline $\mathrm{Cu}$ & 2.67 & 3.561 & 4.033 & 4.517 & 5.012 & 5.518 & 6.034 & 6.559 & 7.094 & 7.638 \\
\hline $\mathrm{Ag}$ & 3.02 & 5.632 & 6.388 & 7.163 & 7.957 & 8.769 & 9.597 & 10.442 & 11.301 & 12.175 \\
\hline $\mathrm{Be}$ & 1.87 & 0.611 & 0.692 & 0.775 & 0.859 & 0.946 & 1.033 & 1.123 & 1.215 & 1.308 \\
\hline $\mathrm{Mg}$ & 2.65 & 2.257 & 2.565 & 2.881 & 3.205 & 3.536 & 3.875 & 4.220 & 4.571 & 4.929 \\
\hline $\mathrm{Cr}$ & 1.86 & 0.599 & 0.678 & 0.759 & 0.842 & 0.927 & 1.013 & 1.101 & 1.191 & 1.282 \\
\hline $\mathrm{Fe}$ & 2.12 & 0.973 & 1.104 & 1.237 & 1.374 & 1.514 & 1.657 & 1.802 & 1.950 & 2.101 \\
\hline $\mathrm{Ni}$ & 2.07 & 0.891 & 1.009 & 1.131 & 1.256 & 1.384 & 1.514 & 1.647 & 1.782 & 1.919 \\
\hline $\mathrm{Zn}$ & 2.31 & 1.343 & 1.524 & 1.710 & 1.900 & 2.095 & 2.294 & 2.496 & 2.703 & 2.913 \\
\hline $\mathrm{Cd}$ & 2.59 & 2.069 & 2.351 & 2.640 & 2.936 & 3.239 & 3.549 & 3.865 & 4.187 & 4.514 \\
\hline $\mathrm{Al}$ & 2.07 & 0.681 & 0.773 & 0.868 & 0.965 & 1.065 & 1.167 & 1.270 & 1.376 & 1.483 \\
\hline $\mathrm{Bi}$ & 2.25 & 1.904 & 2.151 & 2.403 & 2.661 & 2.924 & 3.193 & 3.466 & 3.744 & 4.026 \\
\hline $\mathrm{Ti}$ & 1.92 & 0.512 & 0.581 & 0.652 & 0.725 & 0.800 & 0.876 & 0.953 & 1.032 & 1.112 \\
\hline $\mathrm{Y}$ & 2.61 & 1.649 & 1.876 & 2.110 & 2.350 & 2.595 & 2.846 & 3.102 & 3.362 & 3.628 \\
\hline $\mathrm{Sn}$ & 2.22 & 0.735 & 0.837 & 0.941 & 1.048 & 1.157 & 1.269 & 1.383 & 1.499 & 1.618 \\
\hline $\mathrm{Pb}$ & 2.30 & 0.843 & 0.959 & 1.079 & 1.201 & 1.327 & 1.455 & 1.586 & 1,720 & 1.856 \\
\hline $\mathrm{Mo}$ & 1.61 & 0.164 & 0.186 & 0.209 & 0.233 & 0.257 & 0.282 & 0.307 & 0.332 & 0.359 \\
\hline $\mathrm{W}$ & 1.62 & 0.190 & 0.216 & 0.243 & 0.270 & 0.298 & 0.326 & 0.355 & 0.385 & 0.415 \\
\hline $\mathrm{Au}$ & 2.39 & 0.845 & 0.963 & 1.084 & 1.208 & 1.335 & 1.465 & 1.597 & 1.733 & 1.870 \\
\hline $\mathrm{Pt}$ & 2.00 & 0.493 & 0.561 & 0.630 & 0.701 & 0.774 & 0.848 & 0.924 & 1.002 & 1.0804 \\
\hline $\mathrm{Ta}$ & 2.84 & 1.333 & 1.520 & 1.713 & 1.912 & 2.115 & 2.323 & 2.535 & 2.752 & 2.972 \\
\hline
\end{tabular}


Table 3: Calculated Bulk Modulus and Compressibility of Undeformed Metals and their Experimental values obtained from Kittel (1976)

\begin{tabular}{|l|l|l|l|l|l|}
\hline Metals & $\mathrm{r}_{\mathrm{s}}$ (a.u) & $\begin{array}{l}\text { Cal. Bulk Modulus } \\
\mathrm{Nm}^{-2} \\
\mathrm{x} 10^{11}\end{array}$ & $\begin{array}{l}\text { Exp. Bulk Modulus } \\
\mathrm{Nm}^{-2} \\
\mathrm{x} 10^{11}\end{array}$ & $\begin{array}{l}\text { Cal. Compressibility } \\
{\mathrm{x} 10^{-11} \mathrm{~m}^{2} \mathrm{~N}}^{-1}\end{array}$ & $\begin{array}{l}\text { Exp. Compressibility } \\
\mathrm{x} 10^{-11} \mathrm{~m}^{2} \mathrm{~N}\end{array}$ \\
\hline $\mathrm{K}$ & 4.96 & 0.031 & 0.032 & 32.25 & 31.00 \\
\hline $\mathrm{Cu}$ & 2.67 & 0.322 & 1.37 & 3.10 & 0.73 \\
\hline $\mathrm{Ag}$ & 3.02 & 0.204 & 1.007 & 4.90 & 0.99 \\
\hline $\mathrm{Be}$ & 1.87 & 1.876 & 1.003 & 0.53 & 1.00 \\
\hline $\mathrm{Mg}$ & 2.65 & 0.511 & 0.354 & 1.96 & 2.82 \\
\hline $\mathrm{Cr}$ & 1.86 & 1.914 & 1.901 & 0.52 & 0.53 \\
\hline $\mathrm{Fe}$ & 2.12 & 1.181 & 1.683 & 0.85 & 0.59 \\
\hline $\mathrm{Ni}$ & 2.07 & 1.290 & 1.86 & 0.78 & 0.54 \\
\hline $\mathrm{Zn}$ & 2.31 & 0.857 & 0.598 & 1.17 & 1.67 \\
\hline $\mathrm{Cd}$ & 2.59 & 0.557 & 0.467 & 1.80 & 2.14 \\
\hline $\mathrm{Al}$ & 2.07 & 1.693 & 0.722 & 0.59 & 1.39 \\
\hline $\mathrm{Bi}$ & 2.25 & 0.601 & 0.315 & 1.66 & 3.17 \\
\hline $\mathrm{Ti}$ & 1.92 & 2.247 & 1.051 & 0.45 & 0.95 \\
\hline $\mathrm{Y}$ & 2.61 & 0.700 & 0.366 & 1.43 & 2.73 \\
\hline $\mathrm{Sn}$ & 2.22 & 1.570 & 1.11 & 0.64 & 0.90 \\
\hline $\mathrm{Pb}$ & 2.30 & 1.370 & 0.430 & 0.73 & 2.33 \\
\hline $\mathrm{Mo}$ & 1.61 & 7.042 & 2.725 & 0.14 & 0.37 \\
\hline $\mathrm{W}$ & 1.62 & 6.060 & 3.232 & 0.17 & 0.31 \\
\hline $\mathrm{Au}$ & 2.39 & 1.367 & 1.732 & 0.73 & 0.99 \\
\hline $\mathrm{Pt}$ & 2.00 & 2.339 & 2.783 & 0.43 & 0.36 \\
\hline $\mathrm{Ta}$ & 2.84 & 0.869 & 2.00 & 1.15 & 0.50 \\
\hline
\end{tabular}

\section{Conclusion:-}

In this work, a generalized approach for computing and studying the effect of linear deformation on bulk modulus and compressibility of metals based on the structureless pseudopotential formalism is presented. The results obtained for bulk modulus and compressibility of undeformed metals were in agreement with the experimental values which shows the validity of the model used in the computation. There is high concentration of electron in the high density region than the low density region for both computed and experimental values of the bulk modulus. Metals in the high density region have low compressibility while metals in the low density region have high compressibility. These could be due to the variable electron contribution in the solid and the nature of their electronic state structure. The bulk modulus and compressibility of metals is strongly affected by deformation due to the trend exhibited by different metals during deformation (strain).

\section{References:-}

1. Adesakin, G. E.(2016). Effect of Linear Deformation on Electrical Conductivity of Metal. Advances in Physics Theories and Applications UK. Vol. 53, 10-17.

2. Adeshakin, G.E. and Osiele, O.M. (2012). Surface Energy and Surface Stress of Deformed Metals. Journal of the Nigerian Association of Mathematical Physics, Vol. 21, 421-428.

3. Animalu, A.O.E. (1977).Intermediate quantum Mechanics of crystalline solids. Eagle wood Cliffs, New Jersey.

4. Backofen, W. A. (1972): deformation processing additson - Wesley publishing company. Massachusetts institute of technology.

5. Borg, S.F. (1990): Fundamentals of Engineering Elasticity. Published by World Scientific Publishing Co. Pte. Ltd.

6. Bowen, C., Sugiyama, G. and Alder, B.T. (1974): Static response of the electron gas. Physical Review B. vol. 50, No 20, 14838-14848.

7. Duan, F. and Guojun, J. (2005), Introduction to Condensed Matter Physics. Vol. 1. World scientific publishing Co. Pte. Ltd. P. 199.

8. Elliott, S.R. (1997). The Physics and Chemistry of solids. Trinity College, University of Cambridge UK. Pp 291-308. 
9. Hugh Ford and Alexander, J. M. (1977), Advanced Mechanics of Materials. Publish by Ellis Horwood Limited, Chichester, $2^{\text {nd }}$ Edition. ISBN 85312-056-0 PP 3-5.

10. Iwamoto, N. (1999). Effects of screening on the thermal resistivity of metals due to electron-electron scattering. Physical Review B. 59(15): 9687-9690.

11. Kiejna, A. and Pogosov, V.V. (2000): simple theory of elastic deformed metals: surface energy, stress and work function. Physical Review B. Vol. 62, No 15, 10445-10450.

12. Kiejna, A. and Wojciechowski, K.F. (1996), Metal surface electron physics. Elsevier science. Tokyo, Japan. 293pp.

13. Kittel C. (1976): Introduction to solid state Physics. Fifth edition. John Wiley and sons. Inc. New York.

14. Kiejna A. (1993): Surface properties of simple metals in a structureless pseudopotential model. Physical Review B 47 (12), 7361-7364.

15. Kukkonen, C.A. and Wilkins, J.W. (1979). Electron-Electron Scattering in simple metals. Physical Review B. 19(12): 6075-6093.

16. Ling, D.D and Gelatt, C.D. (1981). Theory of chemical trends in simple metal elastic moduli. Physical Review B.22(2): 557-573.

17. Marder, M.P. (2000). Condensed Matter Physics. John Wiley and sons Inc. New York pp 230-235..

18. Osiele, O.M.and Adesakin G.E and Otobo S.I (2011). Effects of screening on the thermal Resistivity and Compressibility Ratio of metals. Journal of the Nigerian Association of Mathematical Physics, 19, 519-526. Nigeria.

19. Osiele, O.M. and Edema, O.G. (2009). Bulk modulus of metals according to structurelesspseudopotential model. Journal of the Nigerian Association of Mathematical Physics, 14, 197-204. Nigeria.

20. Perdew, J. and Zunger, A. (1981), self-interaction correction to density functional approximation for many electron systems. Physical review B 23(10): 5075-5076.

21. Perdew,J.P, Trans, H.Q, and Smith, E.d. (1990) stabilized jellium structureless pseudopotential model for the cohesive and surface properties of metals. Physical Review B. 18(18): 11627-11636.

22. Pillai, S.D. (2010). Solid State Physics. Published by New Age International (P) Ltd. New Revised Sixth Edition. 4835/24. Ansari Road. Daryaganji. New Delhi - 110002 pp 47-86.

23. Solymar L and Walsh D., (1984): Lectures on the Electrical Properties of Material. Publish by Oxford University Press, New York, United State. $3^{\text {rd }}$ Edition. ISBN 0193511620. Pp98-100.

24. Vacker, J., Hythe, M. and Sumunck, A. (1998). All Electron pseudopotential. Physical Review B. 58(19): 12712-12719. 